\title{
Traumatic spinal epidural hematoma: an alarming and diagnostic challenge in the ED
}

\author{
Krishna Prasad1", Susan Tharian', Jebu A Thomas' \\ 'Department of Emergency Medicine, Pushpagiri Medical College Hospital, Thiruvalla, Kerala, India
}

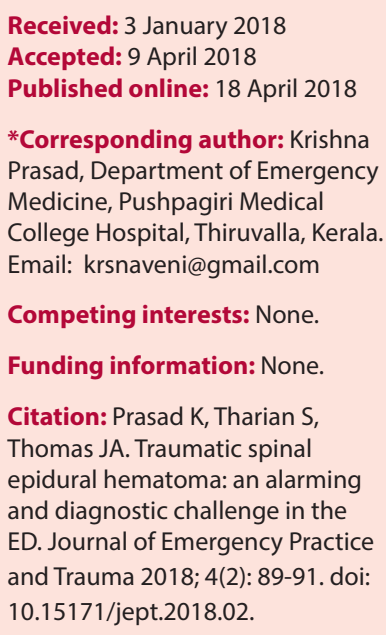

\begin{abstract}
Traumatic spinal epidural hematoma (TSEH) is a neurosurgical emergency which is considered as one of the rare causes of back pain. The onset of symptoms presentation can vary especially following a trauma. Here, we report a case where a young girl had a trivial fall and presented symptoms of back pain three days later. She developed abrupt onset of lower limb weakness during her stay in the emergency department (ED). X-ray and computed tomography (CT) imaging of the spine did not show any obvious abnormality whereas, Magnetic resonance imaging (MRI) imaging revealed hyper-intense lesions in the thoracic spinal segment which led to the diagnosis of TSEH. Our case highlights the importance of the atypical and varied nature of presentation and the choice of investigation which would help in the early diagnosis of this entity.

Keywords: Traumatic spinal epidural hematoma, Back pain, Paraperesis, Emergency department
\end{abstract}

\section{Introduction}

Symptomatic traumatic spinal epidural hematoma (TSEH) is a rare condition described in medical literature (1). Symptomatology of TSEHs can vary from clinically silent to life threatening conditions (2). Because of its rare presentation, the emergency physician should be well versed in knowing its varied presentations, identifying the condition and also be judgmental in advocating MRI imaging of the spine early, thus arriving at a diagnosis quicker which will ultimately lead to better outcome.

\section{Case Report}

A 20-year-old girl was brought to the ED with complaints of severe back pain. She had a history of fall, three days prior at her home. She described that while coming down the staircase; she slipped and fell backwards hitting her back at the edge of a step, following which she got up immediately without any support and with no neurological deficit. She then carried on with her normal day-to-day activities until before going to sleep, she complained of back pain which was mild-to-moderate in nature over her back where she was injured. Her mother reassured her by saying that it will be alright and the pain would subside by the morning. The next day, the pain persisted and she was taken to a local clinic. She underwent X-rays of her spine and was told to be normal. She was discharged giving nonsteroidal anti-inflammatory drugs (NSAIDs) for pain and to review if symptoms persisted. Pain gradually increased and she found symptoms to be increased on sitting. She was able to walk even with pain. The following day, since there was no pain relief with the medications, her parents decided to take her to a local home remedy practitioner who then advised her to undergo ' $k i z h i$ ' over her backbone (traditional massage with medicated poultice). After this procedure, she did not find any relief but her pain aggravated severely. The patient had no similar history before nor was she on any regular medications. On presentation to our ED, the patient was in severe back pain. After giving analgesics, she was examined. On physical examination, she was conscious, oriented and afebrile. Pulse was $94 / \mathrm{min}$, regular and good volume. Blood pressure (BP) was $110 / 80 \mathrm{~mm} \mathrm{Hg}$ in the right arm in supine position. Respiratory rate was 18 breaths/min. The respiratory and cardiovascular system examinations were normal. On neurological examination, higher mental functions and cranial nerves were normal. Gross motor and sensory examination of all 4 limbs were normal. Straight leg raising test was also normal. There was spinous process tenderness over her lower back over T4-T5 region and diffuse tenderness over lumbosacral 
region. Initially X-rays were ordered which did not show any obvious abnormality except for a mild straightening of the lumbar spine. In view of the severe pain and to rule out any trivial fracture, computed tomography (CT) imaging of the lumbar spine was ordered which also showed normal findings. But as soon as the patient was brought back from the CT room, she started complaining that she could not move her lower limbs. This alarmed us and on review examination, there was 0/5 power (Medical Research Council grading) in both the lower limbs with diminished reflexes. There was also complete loss of all sensations in the lower limbs. Next, urgent magnetic resonance imaging (MRI) of the spine was taken which revealed long segment posterior epidural collection from T4 to T10 vertebral level (Figure 1), with hyperintensity noted in the interspinous ligaments (Figure 2) (T2-weighted image and short tau inversion recovery [STIR] sequence). Emergency surgical evacuation of the hematoma was advised by the neurosurgery team but the parents refused treatment as they wanted to take their daughter to another hospital. On follow-up, we came to know she had undergone surgery for hematoma evacuation the next day and had recovered well.

\section{Discussion}

Post-traumatic spinal epidural hematomas are uncommon and account for less than $1 \%$ of all spinal injuries (3). A description by Jackson, in 1869, is credited as the first official record of a spinal epidural hematoma (4). The causes of spinal epidural hematoma can be categorized as spontaneous (most common) and traumatic. Causes of traumatic spinal epidural hematomas (TSEHs) include vertebral fractures, obstetrical birth trauma, lumbar punctures, postsurgical bleeding, epidural anesthesia, and missile injuries. TSEHs can also be present without fractures, but it is very rare (2). Statistically traumatic cases account for a small percentage of all SEH. Common sites for traumatic SEH are the cervicothoracic region and thoracolumbar region (5). The usual clinical presentation for TSEH is dull aching local pain following trauma over the affected region of spine that gradually increases with time and progresses towards paraparesis or quadriparesis, depending on the level of the lesion and nerve root involved (6). MRI is considered as the first choice of investigation and is considered gold standard in the diagnosis of SEH. A CT can be obtained if MRI is not readily available but a negative CT in a high-risk patient should be followed with an MRI (7). Heterogeneous hyperintensity to cord with focal hypointensity on T2-weighted MRI images should suggest the diagnosis of acute spinal epidural hematoma (8). Early surgical intervention is the choice of treatment for traumatic SEH. The procedure includes decompressive laminectomy with removal of the hematoma that may or may not be followed by a laminoplasty (9). The level of preoperative neurologic deficit, severity of the neurologic deficit, and time to operative intervention are the most

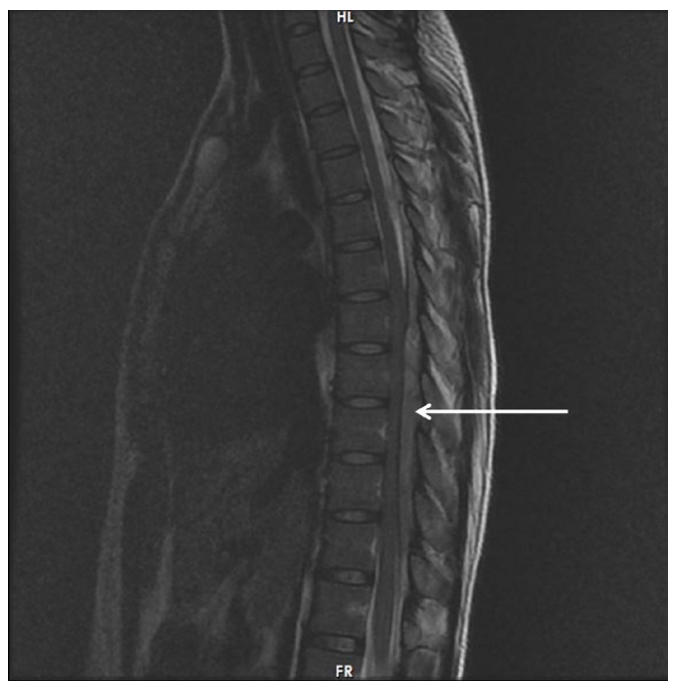

Figure 1. T2-weighted image showing posterior epidural collection from T4 to T10 vertebral level.

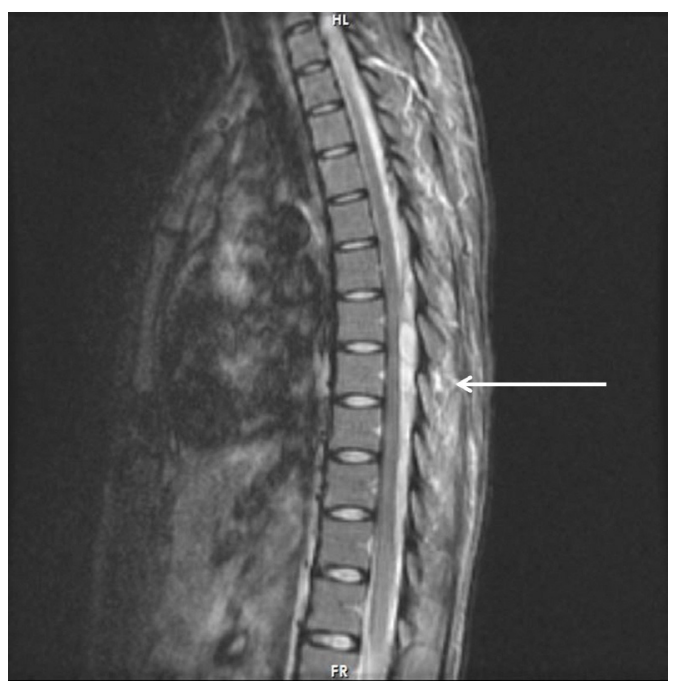

Figure 2. STIR sequence showing hyper-intensity in the interspinous ligaments.

critical factors affecting recovery (10).

\section{Conclusion}

Up to $90 \%$ of low back pain presentations in the ED are due to benign causes. However, there are several important life/limb- threatening diagnoses we must consider in the low back pain patient and most of these diagnoses are easy to miss, especially like the TSEH described in our case. Early MRI whole spine imaging and neurosurgery referral is crucial in the management of TSEH.

\section{Ethical issues}

Written informed consent was obtained from the patient and her mother for publication of this case report.

\section{Author's contributions}

All authors contributed equally to the study. 


\section{References}

1. Boukobza M, Guichard JP, Boissonet M, George B, Reizine D, Gelbert F, et al. Spinal epidural haematoma: report of 11 cases and review of the literature. Neuroradiology 1994; 36(6): 456-9.

2. Bruyn GW, Bosnia NJ. Spinal extraepidural hematoma. In: Vinken PJ, Bruyn GW, eds. Handbook of Clinical Neurology. Injuries of the Spine and Spinal Cord, Part 11.Vol. 26. Amsterdam: North-Holland; 196. p.1-30.

3. Cuenca PJ, Tulley EB, Devita D, Stone A. Delayed traumatic spinal epidural hematoma with spontaneous resolution of symptoms. J Emerg Med 2004; 27(1): 37 41. doi: 10.1016/j.jemermed.2004.02.008.

4. Jackson R. Case of spinal apoplexy. Lancet. 1869; 94(2392): 5-6. doi: 10.1016/S0140-6736(02)67624-X.

5. Liu Z, Jiao Q, Xu J, Wang X, Li S, You C. Spontaneous spinal epidural hematoma: analysis of 23 cases. Surg Neurol 2008; 69(3): 253-60; discussion 60. doi: 10.1016/j. surneu.2007.02.019.

6. Yu HP, Fan SW, Yang HL, Tang TS, Zhou F, Zhao X. Early diagnosis and treatment of acute or subacute spinal epidural hematoma. Chin Med J (Engl) 2007; 120(15): 1303-8.

7. Matsumura A, Namikawa T, Hashimoto R, Okamoto T, Yanagida I, Hoshi M, et al. Clinical management for spontaneous spinal epidural hematoma: diagnosis and treatment. Spine J 2008; 8(3): 534-7. doi: 10.1016/j. spinee.2007.01.009.

8. Fukui MB, Swarnkar AS, Williams RL. Acute spontaneous spinal epidural hematomas. AJNR Am J Neuroradiol 1999; 20(7): 1365-72.

9. Groen RJ. Non-operative treatment of spontaneous spinal epidural hematomas: a review of the literature and a comparison with operative cases. Acta Neurochir (Wien) 2004; 146(2): 103-10. doi: 10.1007/s00701-0030160-9.

10. Garg K, Satyarthee GD, Singla R, Sharma BS. Extensive long-segment cervicothoracic traumatic spinal epidural hematoma with avulsion of $\mathrm{C} 7, \mathrm{C} 8$, and $\mathrm{T} 1$ nerve roots. J Neurosci Rural Pract 2014; 5(4): 414-6. doi: 10.4103/0976-3147.140007. 